Equation of state effects in black hole-neutron star mergers

This article has been downloaded from IOPscience. Please scroll down to see the full text article.

2010 Class. Quantum Grav. 27114106

(http://iopscience.iop.org/0264-9381/27/11/114106)

View the table of contents for this issue, or go to the journal homepage for more

Download details:

IP Address: 131.215.220.165

The article was downloaded on 21/05/2010 at 23:04

Please note that terms and conditions apply. 


\title{
Equation of state effects in black hole-neutron star mergers
}

\author{
Matthew D Duez ${ }^{1}$, Francois Foucart ${ }^{1}$, Lawrence E Kidder ${ }^{1}$, \\ Christian D Ott ${ }^{2}$ and Saul A Teukolsky ${ }^{1,2}$ \\ ${ }^{1}$ Center for Radiophysics and Space Research, Cornell University, Ithaca, NY, 14853, USA \\ 2 Theoretical Astrophysics 350-17, California Institute of Technology, Pasadena, CA 91125, USA \\ E-mail: mduez@astro.cornell.edu
}

Received 18 December 2009, in final form 19 January 2010

Published 10 May 2010

Online at stacks.iop.org/CQG/27/114106

\begin{abstract}
The merger dynamics of a black hole-neutron star (BHNS) binary is influenced by the neutron star equation of state (EoS) through the latter's effect on the neutron star's radius and on the character of the mass transfer onto the black hole. We study these effects by simulating a number of BHNS binaries in full general relativity using a mixed pseudospectral/finite difference code. We consider several models of the neutron star matter EoS, including $\Gamma=2$ and $\Gamma=2.75$ polytropes and the nuclear-theory-based Shen EoS. For models using the Shen EoS, we consider two limits for the evolution of the composition: source-free advection and instantaneous $\beta$-equilibrium. To focus on EoS effects, we fix the mass ratio to $3: 1$ and the initial aligned black hole spin to $a / m=0.5$ for all models. We confirm earlier studies which found that more compact stars create a stronger gravitational wave signal but a smaller postmerger accretion disk. We also vary the EoS while holding the compaction fixed. All mergers are qualitatively similar, but we find signatures of the EoS in the waveform and in the tail and disk structures.
\end{abstract}

PACS numbers: $04.25 \mathrm{D}-$, 04.25.dk, 04.30.Db, 47.75.+f, 95.30.Sf

\section{Introduction}

Black hole-neutron star (BHNS) inspirals and mergers are excellent gravitational wave sources. Also, a BHNS merger may leave a hot, massive accretion disk around the black hole $(\mathrm{BH})$, a promising setup for producing a short-duration gamma-ray burst (GRB). However, reliable predictions regarding the waveform and post-merger state of any specific BHNS merger can only be obtained from fully relativistic numerical simulations.

The merger is strongly affected by the NS equation of state (EoS), i.e. the dependence of the fluid pressure on density, temperature and composition. The EoS determines the radius $R_{\mathrm{NS}}$ of a NS of given mass $M_{\mathrm{NS}}$, and thus it also fixes the star's compaction $\mathcal{C}=M_{\mathrm{NS}} / R_{\mathrm{NS}}$. 
A larger star will be tidally disrupted by the BH's gravity at a larger distance. Also, because the EoS affects how the radius responds to mass loss, it can influence the character of the matter transfer. Stiffer EoS generally have larger $\mathrm{d} R_{\mathrm{NS}} / \mathrm{d} M_{\mathrm{NS}}$, making a star with such an EoS more likely to shrink into its Roche lobe as $M_{\mathrm{NS}}$ decreases, which causes the mass transfer to be more stable. Differences in the stability of mass transfer could conceivably result in qualitative differences in the merger dynamics. The NS EoS at low densities is known, but at high densities it is not well constrained by theory, experiment or observation. It must, therefore, be treated as another parameter in BHNS simulations. If the EoS significantly affects the waveform, then comparison of numerically generated waves with observations may someday provide information about the EoS.

Attempts have been made to estimate the effects of NS EoS in the context of Newtonian BHNS simulations. Newtonian simulations have considered polytropic [1-4], LattimerSwesty [5, 6] and Shen [7-9] EoS. These simulations showed large qualitative differences for different EoS assumptions. For Lattimer-Swesty nuclear matter, the NS disrupts in one mass transfer event and a large post-merger disk is created. For Shen nuclear matter, a NS core can survive multiple mass transfer events and the postmerger disk is much smaller. There are indications that the differences are not quite so dramatic when general relativity (GR) is accounted for. The use of GR-mimicking potentials [10, 11] tends to eliminate episodic mass transfer [12, 13]. Simulations of large mass-ratio cases using the conformally flat approximation of GR also found mass transfer to be less stable (and, therefore, surviving cores to be less likely) than in Newtonian evolutions [14]. These conformally flat-GR studies only considered fairly soft EoS, however.

Published BHNS simulations in full GR have thus-far restricted themselves to modeling NS as $\Gamma=2$ polytropes [15-21]. These have investigated the effects of varying one important EoS-related variable, namely $R_{\mathrm{NS}}[17,18,21]$. Varying $R_{\mathrm{NS}}$ between 12 and $15 \mathrm{~km}$ for a fixed $M_{\mathrm{BH}}$ and $M_{\mathrm{NS}}$, they find, unsurprisingly, that larger stars disrupt farther from the $\mathrm{BH}$, leading to larger disks but weaker merger waveforms. These simulations could not address the importance of the stiffness of the EoS in affecting the mass transfer.

Another important question is whether BHNS mergers eject significant amounts of NS matter (perhaps including $r$-process elements [22, 23]) into the interstellar medium. Newtonian [6], pseudo-Newtonian [12, 13] and conformal-GR [14] simulations predict large ejecta masses, but this has not yet been seen in full GR. However, microphysics in the tidal tail could have important effects. As the density decreases, nucleons recombine into nuclei, heating the gas through the released binding energy. The resulting increase in thermal pressure can strongly affect the tail [9], perhaps helping to unbind material. Material may also be ejected in a neutrino-driven or magnetic field-driven disk wind, effects which are also not captured in current GR simulations.

In this paper, we study the imprint of the NS EoS on the merger dynamics and the resulting waveform and disk. Our investigation is based on simulations of BHNS mergers in full GR carried out with the Cornell-Caltech code SpEC. In a previous paper [19], we demonstrated this code's ability to evolve BHNS inspirals and mergers. Since then, we have made a number of improvements which reduce the constraint violation during merger by an order of magnitude. For this study, we fix the binary mass ratio to $3: 1$ and the initial BH spin to be $\left|\vec{S}_{\mathrm{BH}}\right| / M_{\mathrm{BH}}^{2}=0.5$ (aligned with the orbital angular momentum), and we vary the assumed EoS. We evolve $n=1$ polytropes using a $\Gamma=2$ Gamma-law and $n=4 / 7$ polytropes using $\Gamma=2.75$. We also perform simulations using the tabulated, nuclear-theory-based Shen EoS $[7,8]$. We vary the EoS and the compaction separately to isolate the effect of each.

All our mergers are qualitatively similar: we find no cases of episodic mass transfer, no instances of measurable unbounded outflow, and no cases in which the disruption fails 
to produce a significant disk. There are, however, some quantitative EoS signatures in the waveform above $1 \mathrm{kHz}$ and in the disk. We confirm earlier findings regarding the effects of NS compaction. For a given compaction, stiffer EoS produce larger, longer-lived tidal tails. In every case, the post-merger disk has a mass of $0.05-0.10 M_{\odot}$ and an average temperature of order an $\mathrm{MeV}$.

In section 2, we describe important improvements to our code from [19]. In section 3, we describe the models we evolve. In section 4, we present the results of our evolutions. In section 5, we summarize our findings and consider what remains to be done to accurately sample all of the interesting regions of BHNS parameter space.

\section{Numerical methods}

\subsection{Code improvements}

The main improvements to our code from [19] come from a better allocation of grid points when solving the fluid equations, modified gauge conditions and atmosphere prescriptions. In the discussion below, $g_{a b}$ is the four-metric, $\gamma_{i j}$ is the three-metric, $g=\operatorname{det}\left(g_{a b}\right), N$ is the lapse, $T_{a b}$ is the stress-energy tensor, $\rho$ is the baryonic density, $u^{a}$ is the four-velocity and $v^{i}$ is the fluid three-velocity. Indices $a, b, \ldots$ run from 0 to 3 , while $i, j, \ldots$ run from 1 to 3 . The variables evolved by our hydrodynamics code are the density variable $D=\sqrt{-g} u^{0}$, the energy variable $\tau=N \sqrt{-g} T^{00}-D$ and the momentum variable $S_{i}=\sqrt{-g} T^{0}{ }_{i}$.

Our simulations use two grids [19]: a pseudospectral grid on which Einstein's equations are solved, and a finite difference (FD) for the relativistic fluid equations. For efficient evolutions, the FD grid should adapt to the configuration of the fluid. One approach is to use adaptive mesh refinement, as in some FD-based GR codes [24-26]. We choose instead to modify the mapping between the two grids as soon as a significant flow of matter is approaching the boundary of the FD grid. A translation and rescaling of the coordinates allows the grid to move, grow and shrink so as to follow the fluid evolution. To increase the resolution in the neighborhood of the black hole, we use a map of the form

$$
\begin{aligned}
& r^{\prime}=r\left(r<R_{0}\right) \\
& r^{\prime}=a r^{3}+b r^{2}+c r+d\left(R_{0}<r<R_{1}\right) \\
& r^{\prime}=\alpha r\left(r>R_{1}\right),
\end{aligned}
$$

where $r$ is the coordinate distance to the center of the hole, $R_{0}$ and $R_{1}$ are predetermined length scales, the parameters $(a, b, c, d)$ are chosen so that the map is $C^{1}$ and $\alpha$ varies with the size of the FD grid. (Once the grid becomes large, we maintain a constant resolution in the region $r<R_{0}$ and vary $\alpha$ to fix the location of the outer boundaries.)

The gauge in the generalized harmonic formulation is set by specifying the functions $H_{a}=g^{b c} \Gamma_{a b c}$. During the inspiral, we fix $H_{a}$ in the moving frame, as in our earlier paper [19]. During the merger, we continue to hold $H_{a}$ fixed in the moving frame in the region near the black hole, and we exponentially damp $H_{a}$ to zero in the region far from the hole. We find that fixing $H_{a}$ near the excision zone significantly reduces constraint violations.

As Faber et al [27] have pointed out, the inversion from conservative to primitive hydrodynamic variables is only possible if $S \equiv \gamma^{i j} S_{i} S_{j}<S_{\max } \equiv \tau(\tau+2 D)$. After each evolution step, we impose the condition $S \leqslant S_{\max }^{\text {code }}=f S_{\max }$. We find that $0.99<f<1$ is necessary to avoid causing large effects on the evolution of the tidal tail. Once the values are 'fixed' in this way, primitive variables can be reconstructed, but they may still be unreasonable in very low-density 'atmosphere' regions. Therefore, we next apply limits on the conformal three-velocity $u_{i}$ and the temperature $T$. We emphasize that these limits are only applied to low 
Table 1. Initial data for all runs. $d_{\text {init }}$ is the initial coordinate separation and $\rho_{14}^{\mathrm{c}}$ is the central density of the star in units of $10^{14} \mathrm{~g} \mathrm{~cm}^{-3}$ assuming a star of baryonic mass $M_{b}^{\mathrm{NS}}=1.55 M_{\odot} . e=\frac{B}{\omega d}$ is the eccentricity and $B$ is obtained by fitting the evolution of the trajectory to $\dot{d}=A_{0}+A_{1} t+B \sin (\omega t+\phi)$. $M$ is the ADM mass of the system.

\begin{tabular}{lllllllll}
\hline Case & EoS & $s_{\text {init }}$ & $q$ & $\mathcal{C}_{\text {init }}$ & $\Omega_{\text {init }} M$ & $d_{\text {init }} / M$ & $\rho_{14}^{\mathrm{c}}$ & $e$ \\
\hline$\Gamma 2$ & $\Gamma=2.00$ & 0.5 & 3 & 0.144 & 0.041 & 7.48 & 7.1 & 0.006 \\
$\Gamma 2.75 \mathrm{c} .15$ & $\Gamma=2.75$ & 0.5 & 3 & 0.146 & 0.041 & 7.45 & 4.5 & 0.003 \\
$\Gamma 2.75 \mathrm{c} .17$ & $\Gamma=2.75$ & 0.5 & 3 & 0.173 & 0.041 & 7.42 & 7.6 & 0.014 \\
Shen- $\beta$ & Shen & 0.5 & 3 & 0.147 & 0.041 & 7.59 & 5.0 & 0.004 \\
Shen-Adv & Shen & 0.5 & 3 & 0.147 & 0.041 & 7.59 & 5.0 & 0.004 \\
\hline
\end{tabular}

density regions, several orders of magnitude sparser than the star, the disk, or the tidal tail. We have checked that our evolutions are insensitive to variations in these atmosphere ceilings.

With our standard resolution, the normalized constraint violations peak at $\approx 1 \%$ during mergers. Convergence tests on the $\Gamma=2.75$ case indicate errors in our reported disk masses of $<10 \%$.

\subsection{Use of tabulated $\rho / T / Y_{e}$-dependent EoS}

For composition-dependent EoS, there is a new independent variable to be evolved: the electron fraction $Y_{e}$. Its evolution equation in conservative form is

$$
\partial_{t}\left(D Y_{e}\right)+\partial_{i}\left(D Y_{e} v^{i}\right)=s_{\nu}
$$

where $s_{v}$ is the source term set by weak interactions and neutrino radiation, effects not modeled in our code. Here, we consider two limiting cases. First, we assume that the weak interaction timescales are much longer than the merger timescale. Then we may set $s_{v}=0$ and evolve a continuity equation for $D Y_{e}$. As another limit, we assume that weak interactions act sufficiently quickly to instantaneously enforce $\beta$-equilibrium. Thus, for a given density $\rho$ and $T, Y_{e}$ is set to the value that makes $\mu_{n}=\mu_{p}+\mu_{e}$, where $\mu_{x}$ is the chemical potential of particle $x$. (We assume $\mu_{\nu}$ is negligible.) This effectively removes $Y_{e}$ as a dynamical variable. In neither case do we account for energy loss by neutrino emission. Given that the cooling timescale of the disk will probably not be less than about $0.1 \mathrm{~s}$ (see, e.g. [28]), and our simulations last $\sim 10 \mathrm{~ms}$, ignoring neutrino cooling is reasonable.

We use a tabulated EoS with baryon component taken from Shen et al $[7,8]$ and with lepton and photon contributions added. (See [29] for details.) For low $T(\leqslant 10 \mathrm{MeV})$, the NS EoS is fairly soft $(\Gamma \approx 4 / 3)$ at low $\rho\left(\lesssim 10^{12} \mathrm{~g} \mathrm{~cm}^{-3}\right)$ and stiffer at high $\rho(\Gamma \approx 2.75$ in the Shen model). The Shen EoS predicts a TOV maximum gravitational mass of $2.2 M_{\odot}$.

\section{Cases}

In table 1, we present the initial data for the cases we evolve. For this study, we do not consider the effects of the binary mass ratio $q=M_{\mathrm{BH}} / M_{\mathrm{NS}}$ and set $q=3$ throughout. For each case, we set the NS baryonic mass to $1.55 M_{\odot}$. We wish to study cases that lead to massive disks, and so we include in each case an initial BH spin of $s \equiv\left|\vec{S}_{\mathrm{BH}}\right| / M_{\mathrm{BH}}^{2}=0.5$ orthogonal to the orbital plane. Finally, we neglect the NS spin altogether and focus solely on what is thought to be a good approximation for the most likely scenarios: irrotational stars [30, 31].

To study the effect of compaction, we ran two cases with $\Gamma=2.75$, one with $\mathcal{C}=M_{\mathrm{NS}} / R_{\mathrm{NS}}=0.146$ and one with $\mathcal{C}=0.173$. These runs are labeled ' $\Gamma 2.75 \mathrm{c} .15$ ' and 
' $\Gamma 2.75 \mathrm{c} .17$ ' in the tables and figures below. For our chosen rest mass, the NS gravitational mass is about $M_{\mathrm{NS}}=1.4 M_{\odot}$, so the two compactions correspond to radii of $14.4 \mathrm{~km}$ and $12.1 \mathrm{~km}$.

To separate the effects of the EoS from those of the compaction, we use three equations of state with the same $R_{\mathrm{NS}}(\mathcal{C}=0.15)$. This comparison has not been attempted in previously published full-GR numerical studies. We use two $\Gamma$-law EoS: $\Gamma=2$ (run ' $\Gamma 2$ ') and $\Gamma=2.75$. We also use the tabulated Shen EoS. As mentioned above, we evolve the initial data with Shen EoS in two ways: assuming instantaneous $\beta$-equilibrium (run 'Shen- $\beta$ ') and assuming $s_{v}=0$ (run 'Shen-Adv').

All the initial configurations are generated using our multidomain spectral elliptic solver [32] to solve for quasi-equilibrium configurations of BHNS binaries in the extended conformal thin sandwich formalism [33]. We do not assume conformal flatness, but instead choose a conformal metric approaching Kerr in the neighborhood of the BH [33, 34]. For the polytropic runs, the initial temperature $T_{\text {init }}$ is zero. For the Shen runs, $T_{\text {init }}=0.1 \mathrm{MeV}$, and the initial $Y_{e}$ is set by assuming $\beta$-equilibrium. To ensure that the comparison is not affected by the initial eccentricity of the binary, we also apply the eccentricity removal technique devised by Pfeiffer et al [35] until $e \sim 0.01$. (Without eccentricity removal, we would have $e=0.01-0.06$.)

\section{Results}

\subsection{Qualitative features of the mergers}

For each configuration, the binary passes through 2-3 orbits of inspiral before reaching a coordinate separation of $d \approx 40 \mathrm{~km}(5 M)$, at which point matter starts flowing from the star to the $\mathrm{BH}$. Most of the core of the star is then rapidly accreted onto the hole (within $t \sim 3 \mathrm{~ms}$ $(100 M))$ while a large tidal tail forms. For every EoS, even the stiffest $(\Gamma=2.75)$, the star is disrupted in one extended mass transfer event. This confirms earlier indications that episodic mass transfer does not happen in GR for the realistic range of neutron star EoS. All tails but the one formed by the most compact star extend to distances $d \geqslant 400 \mathrm{~km}$. All of this material, however, remains bound to the system. This result is significant because our Shen-EoS runs do account for recombination effects that occur for $\rho<10^{14} \mathrm{~g} \mathrm{~cm}^{-3}$. It would seem that it is the inclusion of GR, and not inferior microphysics, that causes GR simulations to see no ejecta. (It is, however, possible that some very low-density material is ejected and that this is suppressed in our simulations by the atmosphere prescription.)

Material from the tail falling back toward the hole has enough angular momentum to avoid being immediately accreted: once the core of the star has fallen into the black hole, the accretion rate drops and a disk forms from the remains of the tail. This disk is at first neither axisymmetric nor time independent: the density peaks strongly at the junction of the disk and the tidal tail, and the matter keeps expanding away from the hole. As the accretion rate decreases, a gap opens between the disk and the black hole. At the end of our simulations, the outer disk is still quite nonaxisymmetric, and the tidal tail is settling back onto the disk. The inner disk has a density $\rho \sim 10^{11}-10^{12} \mathrm{~g} \mathrm{~cm}^{-3}$ and temperature $T \sim 0.1-10 \mathrm{MeV}$. It is also relatively thick $\left(H_{\text {disk }} / R_{\text {disk }} \sim 0.1-0.3\right)$.

\subsection{Effects of composition, compaction and stiffness}

The effects of the EoS on the gravitational wave spectrum and on the post-merger disk mass are shown in figures 1 and 2. The leading contribution from the EoS is related to the compaction of the star. As Shibata et al $[18,21]$ found for $\Gamma=2$ stars, we observe that a higher compaction 


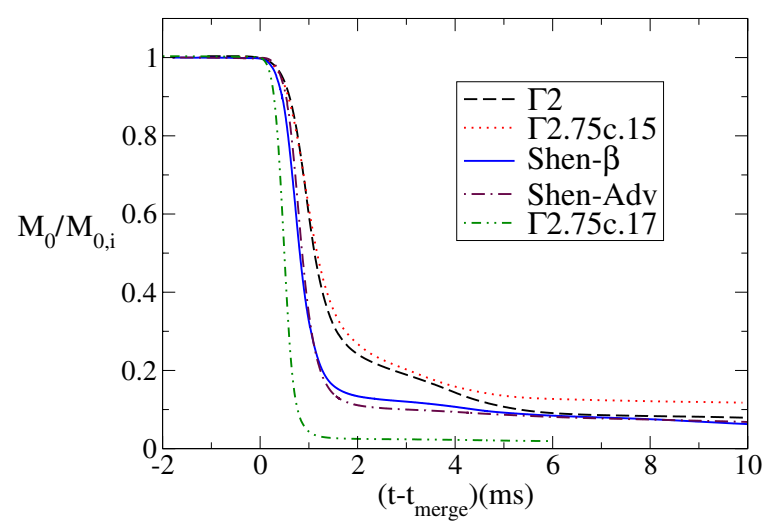

Figure 1. The effect of EoS on the disk mass. $M_{0}$ is the total baryonic mass outside the $\mathrm{BH}$.

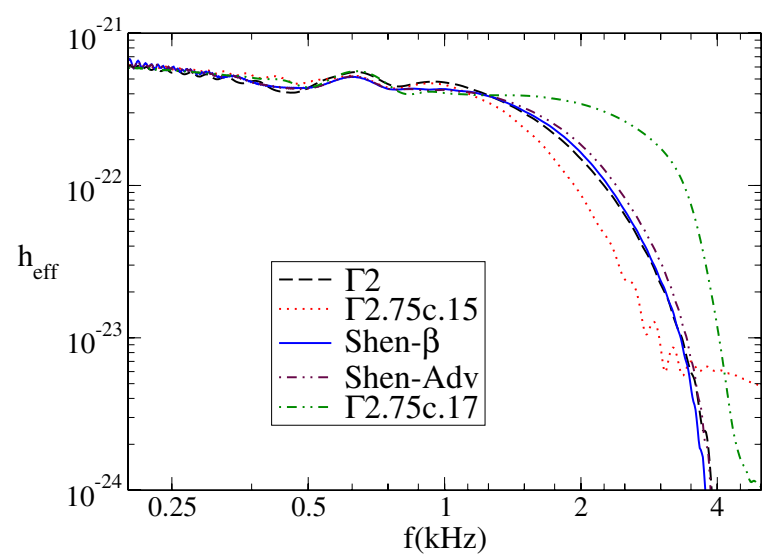

Figure 2. The effective amplitude, as defined in equation (41) of [21], for each run. The assumed distance from the source is $100 \mathrm{Mpc}$.

leads to stronger gravitational waves and a smaller disk. The more compact star reaches the ISCO with minimal distortion and falls nearly entirely into the black hole with only a small fraction of its mass sent out in a relatively short tidal tail extending to $d \sim 250 \mathrm{~km}$. The tail forms a small disk of mass $\approx 0.02 M_{\mathrm{NS}}$. The cutoff frequency of the gravitational waves ( $f \sim 2 \mathrm{kHz})$ is higher than for more extended stars $(f \sim 1 \mathrm{kHz}$ for $\Gamma 2.75 \mathrm{c} .15)$.

By comparison, modifying the EoS while keeping the compaction constant seems to have more modest effects. For NS compaction of $\mathcal{C}=0.15$, every EoS predicts a final disk mass $\sim 0.05-0.1 M_{\mathrm{NS}}$, i.e. $M_{\text {disk }} \approx 0.1 M_{\odot}$. The density-weighted average temperature of the disk is $1-2 \mathrm{MeV}$, although some regions in the disks reach $T \approx 20 \mathrm{MeV}$. Differences in the gravitational wave signal are more visible: the $\Gamma=2.75$ star has a lower cutoff frequency and peak amplitude. But even then, the differences remain smaller than those due to the compactness of the star. The close similarity of the Shen and $\Gamma=2$ waveforms is surprising. A partial explanation may lie in the fact that the Shen-EoS is actually very soft at the densities of the matter after the NS is disrupted. In these regimes, Shen is closer to $\Gamma=2$ than to $\Gamma=2.75$. 
Table 2. The properties of the $\mathrm{BH}$ and accretion torus at time $t=t_{\text {merger }}+8.3 \mathrm{~ms}$. $v_{\text {kick }}$ is the BH kick velocity in $\mathrm{km} \mathrm{s}^{-1} . M_{\text {disk }}$ is the baryonic mass outside of the black hole, $r_{\text {disk }}$ is the radial extent of the disk, $\rho_{\text {disk, } 12}^{\max }$ is the maximum density in units of $10^{12} \mathrm{~g} \mathrm{~cm}^{-3},\langle T\rangle_{\text {disk }}$ is the density-weighted average temperature in $\mathrm{MeV}$ and $\Psi_{4}^{2,2}$ is the amplitude of gravitational waves in the $(2,2)$ mode extracted at $r=75 M$. Note that the disk continues to evolve at late times as $r_{\text {disk }}$ and $\langle T\rangle_{\text {disk }}$ slowly increase.

\begin{tabular}{lllllllll}
\hline Case & $M_{\mathrm{BH}} / M$ & $s_{\text {final }}$ & $v_{\text {kick }}$ & $M_{\text {disk }} / M_{\text {NS }}$ & $r_{\text {disk }} / M$ & $\rho_{\text {disk, }, 12}^{\max }$ & $\langle T\rangle_{\text {disk }}$ & $r M \Psi_{4}^{2,2}$ \\
\hline$\Gamma 2$ & 0.96 & 0.69 & 83 & 0.08 & 12 & 1 & 1.3 & 0.012 \\
$\Gamma 2.75 \mathrm{c} .15$ & 0.94 & 0.69 & 45 & 0.13 & 12 & 1 & 0.6 & 0.010 \\
$\Gamma 2.75 \mathrm{c} .17$ & 0.96 & 0.70 & 75 & 0.02 & 6 & 0.3 & 1.2 & 0.030 \\
Shen- $\beta$ & 0.97 & 0.79 & 63 & 0.07 & 10 & 1 & 2.7 & 0.015 \\
Shen-Adv & 0.97 & 0.80 & 61 & 0.07 & 11 & 1 & 2.5 & 0.016 \\
\hline
\end{tabular}

Another strong effect of the stiffness of the EoS is the different behaviors of the tidal tails, which can lead to important effects on the post-merger disk dynamics. For all EoS, our simulations end with $0.06-0.07 M_{\mathrm{NS}}$ of matter within $200 \mathrm{~km}$ of the $\mathrm{BH}$ - either in the disk or in the process of joining it. The mass and size of the tidal tail, however, vary: for the $\Gamma=2.75$ star, the tail mass is $0.06 M_{\mathrm{NS}}$ and more than $2 \%$ of the matter will eventually go as far as $2000 \mathrm{~km}$ away from the BH before falling back on a timescale of $200 \mathrm{~ms}$ (assuming geodesic motion). When the tail is maximally extended, our grid would be unable to adequately resolve the whole system. Therefore, we stop evolving when the tail is still expanding. On the other hand, the $\Gamma=2$ EoS leads to a much smaller tail, with a mass of $0.02 M_{\mathrm{NS}}$. Only a negligible amount of matter reaches a distance of $500 \mathrm{~km}$, and most of the matter would fall back on the disk within $25 \mathrm{~ms}$ of the disruption. For both polytropes, interactions between the disk and the tail are strong enough to keep the disk from settling to an axisymmetric state over the duration of our simulation. The Shen case also has a tail of mass $0.02 M_{\mathrm{NS}}$, but it is thicker and falls back more rapidly onto the disk. The disk will settle more quickly, and is much closer to axisymmetry at the end of our simulation.

For both Shen- $\beta$ and Shen-Adv runs, the density-weighted average of $Y_{e}$ remains close to $\left\langle Y_{e}\right\rangle=0.09$ throughout the inspiral, so the inspiral is essentially identical for the two runs. The mergers are also similar, although the Shen- $\beta$ waveform decays somewhat more rapidly. The composition of the disk, however, is radically different. For Shen-Adv, the final $\left\langle Y_{e}\right\rangle$ is about 0.09. (It decreases slightly, even though there are no $Y_{e}$ source terms, because the more highly leptonized central region of the NS is swallowed by the BH.) The baryon mass is about $86 \%$ free neutrons, $7 \%$ heavy nuclei, $5 \%$ free protons and $2 \%$ alpha particles. The nuclei have an average $\langle A\rangle \approx 95,\langle Z\rangle \approx 30$. If $\beta$-equilibrium is enforced, $\left\langle Y_{e}\right\rangle$ increases to 0.2 as the NS matter decompresses. The baryon mass is about $65 \%$ free neutrons, $25 \%$ heavy nuclei $(\langle A\rangle \approx 75,\langle Z\rangle \approx 30), 9 \%$ free protons and $1 \%$ alpha particles. The disks produced by the Shen- $\beta$ and Shen-Adv mergers have similar densities and temperatures: the density-weighted average temperature is about $2.5 \mathrm{MeV}$ for Shen-Adv and $2.7 \mathrm{MeV}$ for Shen- $\beta$, although in each case the maximum temperature reaches $\approx 12 \mathrm{MeV}$, and the average is slowly increasing.

\subsection{The final black hole and disk state}

The final state of the black hole is given in table 2 . The final spin $s_{\text {final }}$ is in the range 0.7-0.8, with the Shen runs having higher $s_{\text {final }}$ because they merge somewhat more quickly and have less time to radiate angular momentum. The BH kick velocity is $\sim 10-100 \mathrm{~km} \mathrm{~s}^{-1}$. 


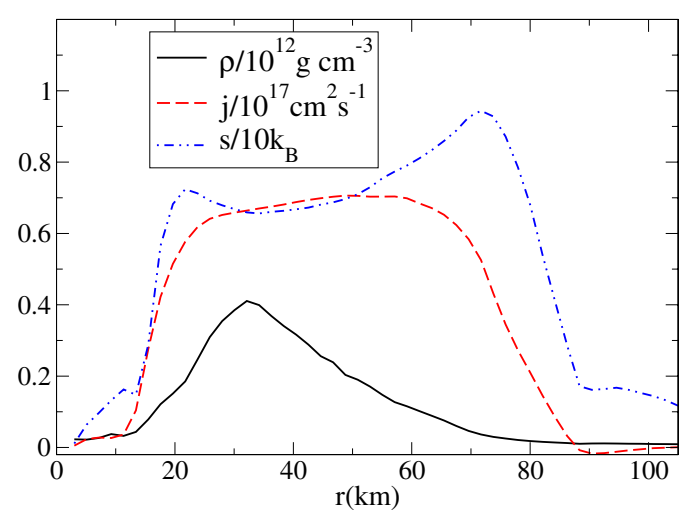

Figure 3. The density $\rho$, specific angular momentum $j$ and specific entropy $s$ of the Shen- $\beta$ disk as a function of cylindrical radius $r$, shown $10 \mathrm{~ms}(370 M)$ after the merger. Each point is a $\rho$-weighted average over the angular and vertical directions.

In figure 3, we plot the profiles of the density $\rho$, specific entropy $s$ and specific angular momentum $j=u_{\phi} / u_{\mathrm{t}}$ for run Shen- $\beta$ at final time $t=10 \mathrm{~ms}$ after the merger. By $3 \mathrm{~ms}$ after merger begins, a distinct torus forms around the BH. By $5 \mathrm{~ms}$ after merger, matter begins to clear out in the region near the hole, and the accretion rate $\dot{M}_{\text {disk }}$ drops to a low value ( $\tau_{\text {acc }}=M_{\text {disk }} / \dot{M}_{\text {disk }} \approx 24 \mathrm{~ms}$ ). The torus has a maximum density of $\rho=10^{12} \mathrm{~g} \mathrm{~cm}^{-3}$ located at a coordinate radius of $r=r_{c}=30 \mathrm{~km}$ from the BH. The width of the torus is $\approx 20 \mathrm{~km}$, and the height is $\approx 10 \mathrm{~km}$. At this time, the disk itself has not yet stabilized. The gas more than $20 \mathrm{~km}$ from the $\mathrm{BH}$ has nearly constant $s$ and $j$, while the gas nearer the hole has somewhat higher $s$ and much lower $j$. Subsequent motions in the fluid lead to modest but positive radial gradients for $s$ and $j$ in most of the high-density region, the exception being the small but negative entropy gradient that persists around $r \approx 30 \mathrm{~km}$. In the still-settling, lower-density outer regions, $s$ and $j$ drop significantly. The angular velocity $\Omega$ decreases with $r$ everywhere: $\Omega \sim r^{-1.2}$ in the high-density region. The assumption of $\beta$-equilibrium is probably not good in the outer regions of the disk, which are optically thin to neutrino emission. The profiles of $\rho$ and $j$ are quite similar for the Shen-Adv disk, so these variables appear to depend weakly on $Y_{e}$.

The longer-term evolution of the disk depends on physical processes not included in these simulations. Since $\mathrm{d} \Omega / \mathrm{d} r<0$, our disks are subject to the magneto-rotational instability (MRI) [36]. Turbulence induced by this instability might have the effect of an $\alpha$-viscosity of magnitude $\alpha \sim 0.01-0.1$ [37]. This will heat the disk, redistribute angular momentum and drive accretion. The disk's only significant cooling mechanism is neutrino emission [38, 39]. 3D Newtonian simulations predict that a neutrino-cooled disk with $\alpha \sim 0.1$ and $M_{\text {disk }}$ similar to ours will accrete on a timescale of $\sim 0.1 \mathrm{~s}$ and release energy in neutrinos at a rate $L_{v} \sim$ $10^{53} \mathrm{erg} \mathrm{s}^{-1}$, possibly making such a merger remnant a viable GRB candidate [40].

\section{Conclusions}

We have investigated the influence of the equation of state on BHNS binaries. We find that the NS compaction has a strong influence on the disk mass and the cutoff frequency of the gravitational waveform. The effects of EoS stiffness for a fixed compaction are weaker. The overall behavior of the merger was found to be independent of EoS stiffness-we never find 
episodic mass transfer or unbound ejecta. The stiffness of the EoS in the lower-density outer layers of the NS does affect the merger dynamics, with stiffer EoS leading to larger and longer-lived tidal tails, and this effect manifests itself in the merger waveform. We find that the evolution of $Y_{e}$ weakly influences the waveform and the final disk mass.

Our simulations suffer from two classes of limitations. First, we have considered only a very small sample of the interesting BHNS parameter space. It is possible that the effects of EoS stiffness are more pronounced at different binary mass ratios or different $\mathrm{BH}$ spins. Also, we have considered only three EoS. A more systematic approach would be to use a single EoS with adjustable parameters (e.g. [5, 41]). An adequate EoS for this purpose would have to cover the range of likely NS stiffness and radius while capturing all of the important physical EoS features, including (for the post-merger evolution) its complicated temperature and composition dependence. Other important areas for improvement involve our treatment of the NS microphysics. To evolve the final disks realistically, the effects of neutrino radiation on the temperature and composition of the matter must be included. The evolution of the disk is also strongly affected by the presence of magnetic fields, and in particular by the MRI. Simulations that include radiation and magneto-hydrodynamics are needed to assess the ability of these disks to produce GRBs.

\section{Acknowledgments}

We thank Evan O'Connor, Harald Pfeiffer and Manuel Tiglio for useful discussions. This work was supported in part by a grant from the Sherman Fairchild Foundation, by NSF grants PHY-0652952 and PHY-0652929 and NASA grant NNX09AF96G. CDO is partially supported through NSF award no AST-0855535. This research was supported in part by the NSF through TeraGrid [42] resources provided by LONI's Queen Bee and NCSA's Ranger clusters. Computations were also performed on the GPC supercomputer at the SciNet HPC Consortium. SciNet is funded by the Canada Foundation for Innovation under the auspices of Compute Canada; the Government of Ontario; Ontario Research Fund-Research Excellence and the University of Toronto. We thank Harald Pfeiffer for getting us access to SciNet by compiling SpEC and submitting our runs there.

\section{References}

[1] Lee W H and Kluzniak W 1999 Astrophys. J. 526178

[2] Lee W H and Kluzniak W 1999 Mon. Not. R. Astron. Soc. 308780

[3] Lee W H 2000 Mon. Not. R. Astron. Soc. 318606

[4] Lee W H 2001 Mon. Not. R. Astron. Soc. 328583

[5] Lattimer J M and Swesty F D 1991 Nucl. Phys. A 535 331-76

[6] Janka H, Eberl T, Ruffert M and Fryer C L 1999 Astrophys. J. Lett. 527 L39-42

[7] Shen H, Toki H, Oyamatsu K and Sumiyoshi K 1998 Nucl. Phys. A 637 435-50

[8] Shen H, Toki H, Oyamatsu K and Sumiyoshi K 1998 Prog. Theor. Phys. 100 1013-31

[9] Rosswog S, Speith R and Wynn G A 2004 Mon. Not. R. Astron. Soc. 3511121

[10] Paczyńsky B and Wiita P J 1980 Astron. Astrophys. 88 23-31

[11] Artemova I V, Bjoernsson G and Novikov I D 1996 Astrophys. J. 461565

[12] Rosswog S 2005 Astrophys. J. 634 1202-13

[13] Ruffert M and Janka H T 2009 arXiv:0906.3998

[14] Faber J A, Baumgarte T W, Shapiro S L, Taniguchi K and Rasio F A 2006 Phys. Rev. D 73024012

[15] Shibata M and Uryu K 2006 Phys. Rev. D 74121503

[16] Shibata M and Uryu K 2007 Class. Quantum Grav. 24 S125-38

[17] Etienne Z B et al 2008 Phys. Rev. D 77084002

[18] Shibata M and Taniguchi K 2008 Phys. Rev. D 77084015

[19] Duez M D, Foucart F, Kidder L E, Pfeiffer H P, Scheel M A and Teukolsky S A 2008 Phys. Rev. D 78104015 
[20] Etienne Z B, Liu Y T, Shapiro S L and Baumgarte T W 2009 Phys. Rev. D 79044024

[21] Shibata M, Kyutoku K, Yamamoto T and Taniguchi K 2009 Phys. Rev. D 79044030

[22] Lattimer J M and Schramm D N 1974 Astrophys. J. Lett. 192 L145-7

[23] Surman R, McLaughlin G C, Ruffert M, Janka H and Hix W R 2008 Astrophys. J. Lett. 679 L117-20

[24] Schnetter E, Hawley S H and Hawke I 2004 Class. Quantum Grav. 21 1465-88

[25] Anderson M, Hirschmann E, Liebling S L and Neilsen D 2006 Class. Quantum Grav. 23 6503-24

[26] Yamamoto T, Shibata M and Taniguchi K 2008 Phys. Rev. D 78064054

[27] Faber J A, Baumgarte T W, Etienne Z B, Shapiro S L and Taniguchi K 2007 Phys. Rev. D 76104021

[28] Lee W H, Ramirez-Ruiz E and Page D 2005 Astrophys. J. 632 421-37

[29] O’Connor E and Ott C D 2009 arXiv:0912.2393

[30] Bildsten L and Cutler C 1992 Astrophys. J. 400 175-80

[31] Kochanek C S 1992 Astrophys. J. 398 234-47

[32] Pfeiffer H P, Kidder L E, Scheel M A and Teukolsky S A 2003 Comput. Phys. Commun. 152 253-73

[33] Foucart F V, Pfeiffer H P, Kidder L E and Teukolsky S A 2008 Phys. Rev. D 77124051

[34] Lovelace G, Owen R, Pfeiffer H P and Chu T 2008 Phys. Rev. D 78084017

[35] Pfeiffer H P et al 2007 Class. Quantum Grav. 24 S59

[36] Balbus S A and Hawley J F 1998 Rev. Mod. Phys. 70 1-53

[37] Shibata M, Sekiguchi Y and Takahashi R 2007 Prog. Theor. Phys. 118 257-302

[38] Popham R, Woosley S E and Fryer C 1999 Astrophys. J. 518 356-74

[39] Chen W and Beloborodov A M 2007 Astrophys. J. 657 383-99

[40] Setiawan S, Ruffert M and Janka H T 2006 Astron. Astrophys. 458 553-67

[41] Read J S et al 2009 Phys. Rev. D 79124033

[42] Catlett C et al 2007 Advances in Parallel Computing ed L Grandinetti (Amsterdam: IOS Press) 